\title{
O estudo da rigidez em lajes utilizando o parâmetro lambda
}

\author{
The study of stiffness in slabs using the lambda parameter \\ La investigación de la rigidez en losas utilizando el parâmetro lambda
}

L'étude de la rigidité chez les dalles en utilisant le paramètre lambda

\author{
Gilson de Sousa Oliveira \\ Neto (OrcII) \\ gilsonsneto@gmail.com \\ Universidade de Fortaleza - \\ Unifor
}

Osmar Alexandre do

Amaral Neto (OrcID)

osmaralexandre22@hotmail.

com

Universidade de Fortaleza -

Unifor

Vera Lúcia da Silva (OrcID) vera@unifor.com

Universidade de Fortaleza Unifor

\section{Eduardo Cesar Cordeiro}

Leite (OrcID)

eduardoleite@unifor.com

Universidade de Fortaleza -

Unifor

\section{Ítalo Linhares Salomão}

(OrcII)

italosalomao@unifor.com.br Universidade de Fortaleza Unifor

\begin{abstract}
Resumo
O cálculo de lajes pode ser realizado através de processos simplificados, tendo como o parâmetro lambda $(\lambda)$ o dado inicial a ser determinado. Esse valor é constituído pela razão entre o lado menos rígido $\left(1_{y}\right)$ e o lado mais rígido $\left(1_{x}\right)$. Tendo como base a Tabela de Marcus, na maioria dos casos, a viga com menor comprimento é a mais rígida, porém isso pode variar em três exceções, quais sejam: laje constituída por uma faixa com dois apoios simples e outra perpendicular com um apoio simples e um engastado (caso 2); laje composta por uma faixa com dois apoios simples e outra perpendicular com dois apoios engastados (caso 4); laje formada por uma faixa com um apoio simples e o outro engastado e uma segunda faixa com dois apoios engastados (caso 5). Para a determinação do elemento de maior rigidez de forma simplificada, foram criados parâmetros a partir de métodos estatísticos gerados em função do deslocamento para cada tipo de faixa representada por viga. A amostra escolhida foi de 600 vigas, sendo 200 para cada um dos 3 casos. O coeficiente para definição do elemento de maior rigidez determinado para o caso 2 foi de 0,810975667 , tem-se 0,675694 para o caso 4 e para o caso 5 obteve-se 0,839303 para o caso 5 . Além disso, podem-se perceber as particularidades para cada caso e o comportamento do gráfico de deslocamento, que varia para cada tipo de viga.
\end{abstract}

Palavras-chave: Laje. Parâmetro lambda. Rigidez.

\begin{abstract}
Slab design can be calculated through simplified methods, being the lambda parameter $(\lambda)$ the initial data to be determined. This value consists of a ratio between the less rigid side (ly) and the stiffer side (lx). Based on the Marcus Chart, in most cases, the beam with the shortest length is the most rigid, however, this can vary in three exceptions, which are: a slab consisting of a linear region with two pinned supports and the other one perpendicular with a pinned support and a fixed one (Case 2); a slab composed of a linear region with two pinned supports and the one perpendicular with two fixed supports (Case 4); a slab formed by a linear region with a pinned support and the other one fixed and a second linear region with two fixed supports (Case 5). For the simplified determination form of the stiffer element, parameters were created from statistical methods generated as a function of the displacement for each type of linear region represented as a beam. 600 beams were chosen as a sample, 200 for each of the 3 types. The coefficient for lambda $(\lambda)$ of case 2 was 0.810975667 , for case 4 was 0.675694 and for case 5 was 0.839303 . In addition, one can see the particularities for each case and the behavior of the displacement graph, which varies for each type of beam.
\end{abstract}

Keywords: Slab. Parameter lambda. Stiffness.

\section{Resumen}

El cálculo de losas puede ser realizado por medio de procesos simplificados, teniendo como parámetro lambda $(\lambda)$ el dato inicial a ser determinado. Este valor es construido por la división entre el lado menos rígido ( $1_{\mathrm{y}}$ ) y el lado más rígido $\left(\mathrm{l}_{\mathrm{x}}\right)$. Teniendo como base la Tabla de Marcus, en la mayoría de los casos, la viga con menor longitud es la más rígida, sin embargo eso puede variar en tres excepciones que son: losa constituida por una banda con dos soportes simple y otra perpendicular con un soporte simple y 
un engastado (Caso 2); losa compuesta por una banda con dos soportes simple y otra perpendicular con dos soportes engastados (Caso 4); losa formada por una banda con un soporte simple y el otro engastado y una segunda banda con dos soportes engastados (Caso 5). Para la determinación del elemento con rigidez más grande de forma simplificada, se crearon parámetros a partir de métodos estadísticos generados en función del desplazamiento para cada tipo de banda representada por viga. La muestra elegida fue la de 600 vigas, siendo 200 para cada uno de los 3 casos. El coeficiente para definición del elemento de rigidez más grande determinado para el caso 2 fue de 0,810975667 , para el caso 4 tenemos 0,675694 y para el caso 5 obtuvimos 0,839303 . Además de eso, se pudo notar las particularidades para cada caso y el comportamiento del gráfico de desplazamiento, que cambia para cada tipo de viga.

Palabras-clave: Losa. Parámetro lambda. Rigidez.

\begin{abstract}
Résumé
Le calcul des dalles peuvent être exécutées par des procédures simplifiées, en prenant comme donnée initiale à être déterminé, un paramètre lambda $(\lambda)$. Cette valeur est le rapport entre le côté moins rigide (1y) et le côté plus rigide (1x). Si on prend comme base la Table de Marcus, dans la plupart des cas, la poutre plus courte est la plus rigide, néanmoins, cela peut varier de trois exceptions: si la dalle se compose d'une piste avec deux supports simples et une autre perpendiculaire avec un support simple et un serti (cas 2); si la dalle est composée d'une piste avec deux supports simples et une autre perpendiculaire avec deux supports sertis (cas 4); si la dalle est formée par une bande avec un support simple et l'autre support serti plus une seconde piste avec deux support sertis (cas 5). Pour déterminer de façon simplifiée l'élément avec la rigidité supérieure, certains paramètres ont été établis à partir des méthodes statistiques générées selon le déplacement pour chaque type de bande représentée par poutre. L'échantillon choisi a été 600 poutres, c'est-à-dire, 200 pour chacun des 3 cas. Le coefficient pour définir l'élément avec la plus grande rigidité donné dans le cas 2 a été 0.810975667 , dans le cas 4 a été 40.675694 et dans le cas 5 on a obtenu 0.839303 . En outre, on peut percevoir les particularités de chaque cas et le comportement de la courbe de déplacement, qui varie pour chaque type de poutre.
\end{abstract}

Mot-Clés: Dalle. Paramètre lambda. Rigidité.

\title{
Considerações iniciais
}

Segundo Bastos (2015), as lajes são projetadas para receber a maior parte das ações aplicadas em uma construção e em sua utilização, sendo comumente oriundas de pessoas, móveis, pisos e paredes, além dos diferentes tipos de carga que podem existir em função da arquitetura do espaço que a laje faz parte. As ações são, normalmente, perpendiculares ao seu plano, podendo ser partilhadas na sua área, distribuídas linearmente ou como forças concentradas. Embora menos banal, também podem ocorrer ações externas na forma de momentos fletores, normalmente aplicados nas bordas das lajes.

As lajes são classificadas como elementos planos bidimensionais, que são aqueles onde duas dimensões, o comprimento e a largura, são da mesma ordem de grandeza e muito maiores que a terceira dimensão, a espessura. As lajes são também chamadas de elementos de superfície, ou placas (BASTOS, 2015, p. 1).

Esses elementos, quando submetidas aos carregamentos externos, deformam, surgindo os esforços internos para manter o equilíbrio da peça. Surge, portanto, a necessidade da determinação desses esforços para a realização do dimensionamento da laje de forma segura, econômica e duradoura. Devido à complexidade de sua determinação, é habitual a criação de métodos simplificados, a fim de tornar viável a realização dos cálculos. Dentre os diversos processos, tem-se, de forma mais usual, o método de Marcus.

O método de Marcus consiste na subdivisão da laje em faixas nos sentidos X e Y, sendo calculados os esforços internos nas faixas principais em cada um dos eixos. Esse método difere de outros pela consideração, através de coeficientes, da interação entre as faixas e dos momentos de torção gerados por esse fato. Para o cálculo através desse método, é necessária a utilização de uma tabela desenvolvida, em que o dado de entrada é a relação do vão menos rígido e do mais rígido, formando o coeficiente lambda. É, portanto, de suma importância a distinção da rigidez entre os vãos. Marcus divide as lajes em dez possíveis casos, conforme apresentado na figura 01. 
Figura 1 - Tipos de laje em função dos vínculos e de acordo com o Marcus.
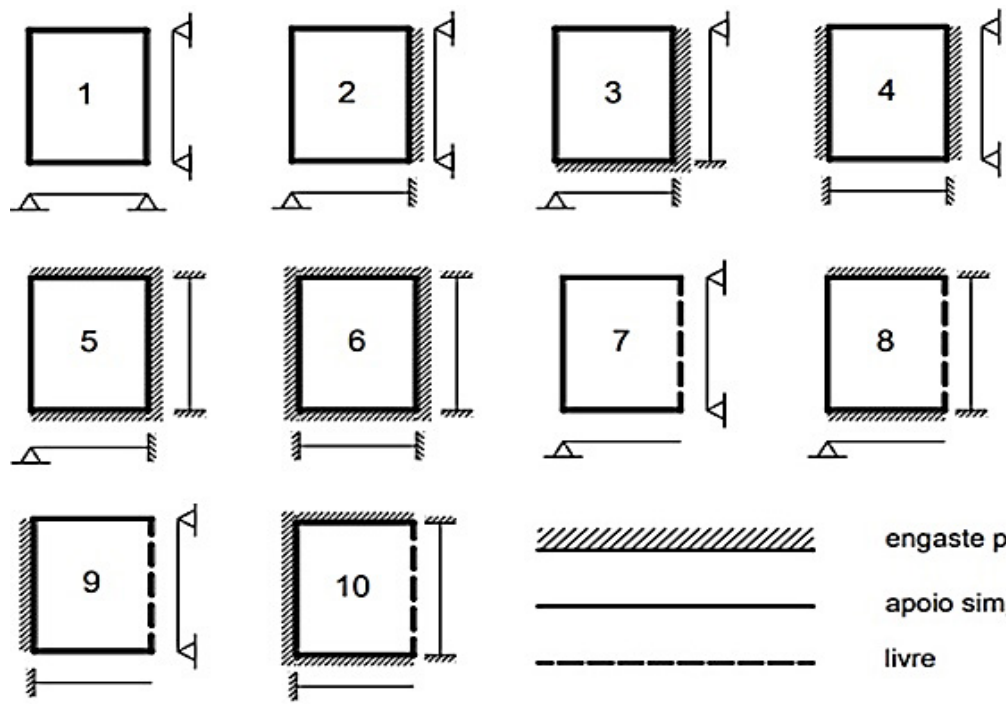

engaste perfeito

apoio simples

livre

Fonte: Os autores (2017).

O estudo da rigidez de elementos pode ser baseado no deslocamento a que a estrutura está submetida, ou seja, quando se deseja determinar, dentre dois elementos, qual apresenta maior rigidez, é possível sua deliberação a partir daquele com o menor deslocamento. Por sua vez, o cálculo dos deslocamentos ocorre, dentre os diversos métodos, através do método de Lagrange ou método energético.

Os métodos energéticos ocorrem mediante a deformação do material, que acontece, por sua vez, quando se tem cargas aplicadas a um corpo. De forma que nenhuma energia seja perdida sob meio de calor, o trabalho externo realizado pelas cargas será convertido em trabalho interno, conhecido como energia de deformação. Essa energia se demonstra sempre de forma positiva, armazenada no corpo e causada pelo efeito da tensão normal ou da tensão de cisalhamento.

O princípio dos trabalhos virtuais afirma que, se uma partícula, um corpo rígido ou, de maneira mais geral, um sistema de corpos ligados entre si, em equilíbrio sob a ação de várias forças externas, sofre um deslocamento arbitrário a partir dessa posição de equilíbrio, o trabalho total efetuado pelas forças externas durante o deslocamento é nulo. (BEER, 2013, p. 560)

A partir da referência supracitada, tem-se que, quando uma partícula está em equilíbrio, o trabalho virtual total das forças operantes é zero, seja qual for o deslocamento virtual dessa partícula (BEER et al., 2013).

Para Hibbler (2014), sempre que se impede um corpo da realização de movimento, as cargas devem atender às condições de equilíbrio, e os deslocamentos às de compatibilidade. As condições de equilíbrio determinam que as cargas internas estejam relacionadas apenas com as cargas externas, e as condições de compatibilidade determinam que os deslocamentos internos fiquem relacionados unicamente com as deformações internas.

De forma a tornear as limitações, aplica-se uma força imaginária P' sobre um corpo no ponto A, tal que P' aja na mesma direção que o deslocamento $(\Delta)$. Ademais, antes da aplicação das cargas reais, a carga é aplicada ao corpo. Por conveniência, atribui-se uma intensidade unitária $\mathrm{P}^{\prime}=1$. Dessa forma, considerando apenas a conservação de energia virtual, o trabalho virtual externo é igual ao trabalho virtual interno. Sendo assim, pode-se escrever a equação (1) do trabalho virtual como:

$$
1 . \Delta=\Sigma \mathrm{u} \cdot \mathrm{dL}
$$

$\mathrm{P}^{\prime}=1$ = carga virtual externa unitária que age na direção de

$u=$ carga virtual interna que age sobre o elemento

$\Delta=$ deslocamento externo provocado pelas cargas reais

$\mathrm{dL}=$ deslocamento interno do elemento na direção de $\mathrm{u}$, provocado pelas mesmas cargas reais 
Segundo Hibbler (2014), de forma similar, para determinar a inclinação da tangente em um ponto sobre o corpo ou o deslocamento rotacional, aplica-se um momento virtual M' de intensidade unitária. Dessa forma, pode-se determinar pelo trabalho virtual a seguinte equação (2):

$$
1 . \theta=\Sigma \mathrm{u} \theta \mathrm{Dl}
$$

$\mathrm{M}^{\prime}=1=$ momento unitário virtual externo que age na direção de $\theta$

$u \theta=$ carga virtual interna que age sobre um elemento

$\theta=$ deslocamento rotacional em radianos provocado pelas cargas reais

$\mathrm{dL}=$ deslocamento interno do elemento na direção de $u \theta$ provocado pelas cargas reais

Mediante a determinação dos deslocamentos nas faixas das lajes, é necessário, para a obtenção de valores a serem utilizados para projetos estruturais, a utilização de um estudo estatístico, tornando os resultados confiáveis.

O Box Plot (também chamado de box e whisker plot) é um método alternativo ao histograma e ao ramoe-folha para representar os dados. O Box Plot fornece informação sobre as seguintes características do conjunto de dados: localização, dispersão, assimetria, comprimento da cauda e outliers (medidas discrepantes) (ESCOLA EDTI, 2017).

Dessa forma, o gráfico Box Plot pode ser interpretado de acordo com a figura 02, em que as linhas verticais exibidas representam os valores máximos e mínimos da distribuição de dados. O retângulo inferior mostra o valor do quartil 1; o superior, do quartil 3; e a linha branca horizontal do meio, a mediana. Com isso, pode-se observar a distribuição dos dados analisados, em torno da mediana.

Figura 2 - Interpretação do Box Plot

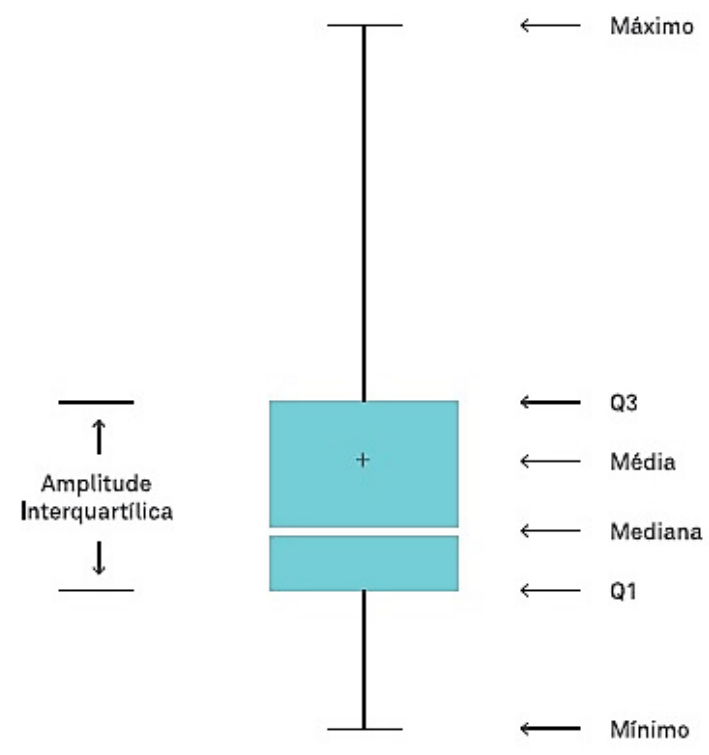

Fonte: Escola Edti, 2017

Para o melhor entendimento do método utilizado, é preciso basear-se na "Regra do evento raro para interferência estatística", a qual estabelece: caso a probabilidade de um evento observado específico ocorrer for mínima sob determinada suposição, pode-se concluir que a hipótese possivelmente é incorreta. Direcionando por essa regra, deve-se testar uma teoria a partir da análise dos dados da amostra na tentativa de diferenciar os resultados que podem ocorrer por acaso e os que são improváveis de acontecer por acaso. Pode-se explicar a ocorrência dos resultados improváveis supondo que o evento raro realmente aconteceu ou que a suposição adjacente não é verdadeira.

Nesse contexto, a estimação média populacional é um exemplo de teste de hipótese e, para seu melhor entendimento, faz-se necessário saber a definição dos seguintes parâmetros: estimativa pontual intervalo de confiança, nível de confiança, 
valor crítico e margem de erro. Além disso, para o uso bem-sucedido desse método, deve-se ter conhecimento de três conceitos determinados por Triola (2014) e expressos a seguir:

- A média amostral é a melhor estimativa pontual da média populacional $\mu$.

- Saber como deve interpretar os intervalos de confiança e como usar dados amostrais na construção de tal intervalo para estimar a média populacional.

- Determinar o tamanho da amostra necessário para estimar a média populacional.

Pode-se definir estimativa pontual como um valor específico utilizado a fim de aproximar uma variável populacional. Diferente de outros métodos, a usualidade da estimativa pontual se altera e, com isso, para melhorar o cálculo da média populacional $\mu$ tem-se a média amostral . Assim, torna-se um estimador não visado desse parâmetro $\mu$. Desse modo, para muitas populações, as médias amostrais tendem a se diversificar menos do que as outras medidas centrais, de forma que a média amostral é, usualmente, a melhor estimativa pontual que a média populacional $\mu$, contudo ela não indica o quão preferível é. Obtêm-se melhores informações através do intervalo de confiança, que consiste em uma faixa de valores (TRIOLA, 2014).

Conceitualmente, o intervalo de confiança, também conhecido por IC, é um período de resultados utilizados para supor o real valor do parâmetro populacional. Essa estimativa consiste em um intervalo de possíveis valores, ao invés de um só, ela melhor estipula a verdadeira medida em comparação a estimativa pontual. No entanto, para o cálculo do intervalo de confiança, é necessário estimar um grau de confiabilidade dos dados obtidos e, com esse objetivo, foi criado o nível de confiança (TRIOLA, 2014).

O nível de confiança é a probabilidade 1- $\alpha$ (em geral, expressa como o valor equivalente em porcentagem) de que o intervalo de confiança realmente contenha parâmetro populacional, supondo que o processo de estimação seja repetido um grande número de vezes. (O nível de confiança é também chamado de grau de confiança ou coeficiente de confiança). (TRIOLA, 2014, p. 267).

Dessa forma, o nível de confiança fornece a taxa de sucesso no processo usado na construção do intervalo de confiança. As taxas mais usuais são: $90 \%(\alpha=0,1), 95 \%(\alpha=0,05)$ e $99 \%(\alpha=0,01)$. Neste trabalho acadêmico, foi escolhida a taxa de $95 \%$, pois, além de ser a mais comum, fornece bom equilíbrio entre a precisão (conforme refletido na largura do intervalo de confiança) e a confiabilidade (conforme expresso no nível de confiança) (TRIOLA 2014).

Por fim, tendo como finalidade estimar o intervalo de confiança, deve-se estimar a média populacional. Para isto, têm-se três pré-requisitos:

- A amostra é uma amostra aleatória simples

- O valor do desvio padrão populacional $\sigma$ é conhecido.

- Se uma ou ambas das condições são satisfeitas: a população é normalmente distribuída ou n $>30$.

Após atender os pré-requisitos, pode-se calcular o intervalo de confiança pela equação (3):

IC: $\left[\bar{x}-\frac{Z(1-\alpha)}{2} \cdot \frac{\sigma}{\sqrt{n}} \leq \mu \leq \bar{x}+\frac{Z(1-\alpha)}{2} \cdot \frac{\sigma}{\sqrt{n}}\right]=(1-\alpha)$

ou

$\bar{x}-E \leq \mu \leq \bar{x}+E ;$ onde $E=\frac{Z(1-\alpha)}{2} \cdot \frac{\sigma}{\sqrt{n}}$

Onde:

$\mu=$ média populacional

$\sigma=$ desvio padrão populacional

$\bar{x}=$ média amostral

$\mathrm{n}=$ número de valores amostrais

$Z_{(1-\alpha)}=$ escore z que separa uma área de $\alpha / 2$ na cauda direita da distribuição normal padrão

$\mathrm{E}=$ margem de erro 


\section{Metodologia}

A metodologia utilizada tem como propósito determinar a faixa, dentre as definidas no eixo x e y, que representa o sentido mais rígido de uma laje. Foi, inicialmente, calculado o deslocamento para os três casos em análise, de forma individual e com diferentes comprimentos, sendo eles 2, 4 e 5 da tabela de Marcus.

Para realizar o estudo de cada viga, é necessário definir as leis constitutivas e as condições de contorno, ou seja, o apoio e a carga aplicada na estrutura. Dessa forma, é possível medir o deslocamento e, com isso, formar uma tabela para cada caso, composta pelo comprimento e o deslocamento.

Com o intuito de definir um coeficiente que possa determinar o sentido mais rígido, determinam-se os comprimentos através do vão efetivo de cada eixo da laje. Assim, foram adotadas distâncias que variam de ordem crescente, com intervalo de $0,1 \mathrm{~m}$ a $20 \mathrm{~m}$, e carregamentos unitários de $1 \mathrm{kN} / \mathrm{m}$. Posteriormente, fez-se necessária a determinação das condições de apoio, de forma a compor os dados para os casos 2, 4 e 5 do método de Marcus, conforme mostrado na figura 03 de forma isolada por eixo.

Figura 3 - Tipos de apoio em análise

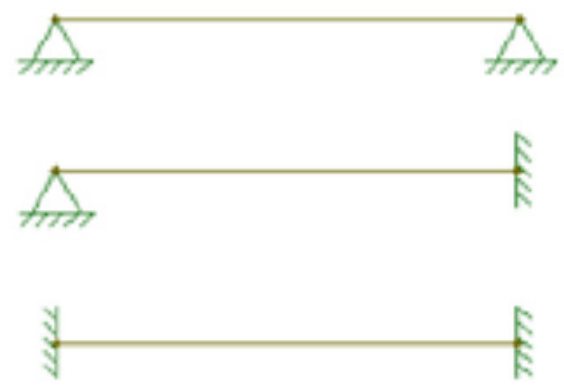

Fonte: Os autores (2017).

Desse modo, foram dimensionadas 200 vigas para cada um dos casos, gerando uma amostra de 600 modelos. Após a análise, foram computados os valores dos deslocamentos que variam de acordo com o tamanho da viga e do tipo de apoio.

A partir dos dados obtidos, foi elaborada a tabela 01 onde, na primeira coluna, está representado os comprimentos das barras, e as três colunas restantes são compostas pelos deslocamentos para os respectivos apoios e comprimentos. Esta tabela mostra uma parcela dos resultados encontrados para os três casos de apoio.

Tabela 1 - Tabela comparativa dos deslocamentos em metro

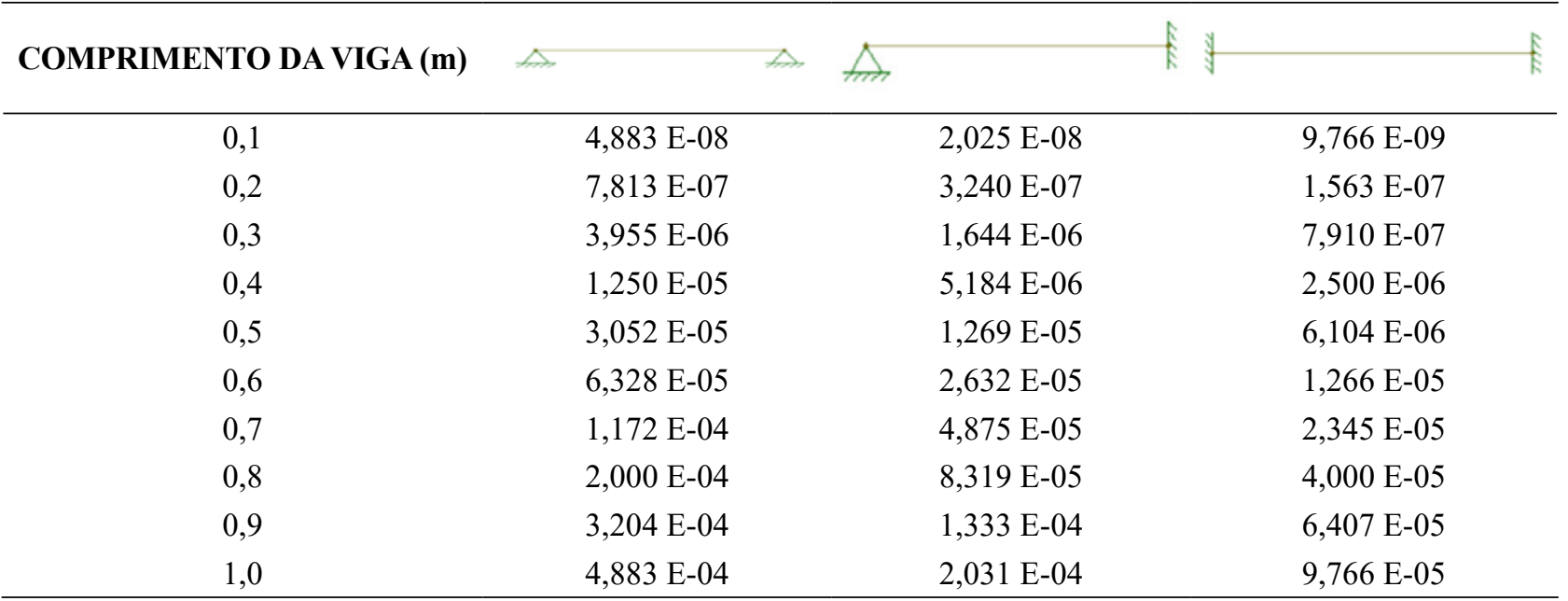

Fonte: Os autores (2017). 
Na sequência, foram desenvolvidas três tabelas, elaboradas a partir dos deslocamentos apresentados na tabela 1. Sua composição foi feita para cada caso do método de Marcus em análise e teve como objetivo a obtenção do fator 1 que explicitasse ao calculista o ponto de inversão de rigidez, isto é, a partir de qual relação a faixa que visualmente tem a menor rigidez, passa a ser mais rígida do que a faixa que visualmente tem maior rigidez.

A primeira coluna das tabelas 2, 3 e 4 ilustra, de forma ordenada crescente, o comprimento de um tipo de faixa. Já a segunda coluna ilustra a extensão da faixa perpendicular a esta, da mesma laje retangular, que tenha o menor deslocamento mais próximo do deslocamento da faixa da primeira coluna. Por fim, a terceira coluna $(\lambda)$ apresenta a divisão entre os comprimentos. Após o término dessas tabelas, notou-se que há uma enorme variação entre os coeficientes calculados. Essa inconstância se dá pela limitação dos dados coletados, tornando-se maior para os menores comprimentos. Portanto, para atingir o objetivo de estabelecer um coeficiente, é necessário realizar um estudo estatístico dos dados, a fim de propor esse parâmetro com maior precisão.

Desse modo, como forma de originar um coeficiente para cada caso, foi realizado um estudo estatístico com a finalidade de determinar o intervalo de confiança no qual atuam os coeficientes estudados.

Tabela 2 - Tabela com os coeficientes $\boldsymbol{l}$ para o caso 2

\begin{tabular}{ccc}
\hline \multicolumn{1}{c}{ Ant } & & $\mathbf{I}$ \\
\hline 0,1 & 0,1 & 1 \\
0,2 & 0,2 & 1 \\
0,3 & 0,3 & 1 \\
0,4 & 0,4 & 1 \\
0,5 & 0,6 & 0,833333 \\
0,6 & 0,7 & 0,857143 \\
0,7 & 0,8 & 0,875 \\
0,8 & 0,9 & 0,888889 \\
0,9 & 1,1 & 0,818182 \\
1 & 1,2 & 0,833333 \\
\hline
\end{tabular}

Fonte: Os autores (2017).

Tabela 3 - Tabela com os coeficientes $\boldsymbol{l}$ para o caso 4

\begin{tabular}{|c|c|c|}
\hline$\Rightarrow$ & 为 & 1 \\
\hline 0,1 & 0,1 & 1 \\
\hline 0,2 & 0,2 & 1 \\
\hline 0,3 & 0,4 & 0,75 \\
\hline 0,4 & 0,5 & 0,8 \\
\hline 0,5 & 0,7 & 0,714286 \\
\hline 0,6 & 0,8 & 0,75 \\
\hline 0,7 & 1 & 0,7 \\
\hline 0,8 & 1,1 & 0,727273 \\
\hline 0,9 & 1,3 & 0,692308 \\
\hline 1 & 1,4 & 0,714286 \\
\hline
\end{tabular}

Fonte: Os autores (2017). 
Tabela 4 - Tabela com os coeficientes $\boldsymbol{l}$ para o caso 5

\begin{tabular}{ccc}
\hline & 杖 & I \\
\hline 0,1 & & 1 \\
0,2 & 0,1 & 0,666667 \\
0,3 & 0,3 & 0,75 \\
0,4 & 0,4 & 1 \\
0,5 & 0,4 & 1 \\
0,6 & 0,5 & 0,857143 \\
0,7 & 0,7 & 0,875 \\
0,8 & 0,8 & 0,888889 \\
0,9 & 0,9 & 0,9 \\
1 & 1 & 0,833333 \\
\hline
\end{tabular}

Fonte: Os autores (2017).

\section{Resultados e discussão}

A fim de criar o valor do coeficiente 1 que forneça ao engenheiro, de forma clara e concisa, qual faixa da laje apresenta maior rigidez - e com os valores dos 600 modelos gerados e estudados, conforme apresentado nas tabelas 1 a 4 -, foi calculada, para o caso 2, a estatística descritiva, conforme expressa na tabela 5 e na figura 4 .

Tabela 5 - Estatística descritiva caso 2

\begin{tabular}{cc}
\hline Média & 0,810975667 \\
\hline Erro padrão & 0,000967269 \\
Mediana & 0,807228916 \\
Modo & 0,8125 \\
Desvio padrão & 0,012081183 \\
Variância da amostra & 0,000145955 \\
Curtose & 17,81237206 \\
Assimetria & 3,852640302 \\
Intervalo & 0,085739283 \\
Mínimo & 0,803149606 \\
Máximo & 0,888888889 \\
CV & $1,490 \%$ \\
Contagem & 156 \\
\hline
\end{tabular}

Fonte: Os autores (2017) 
Figura 4 - Box Plot caso 2

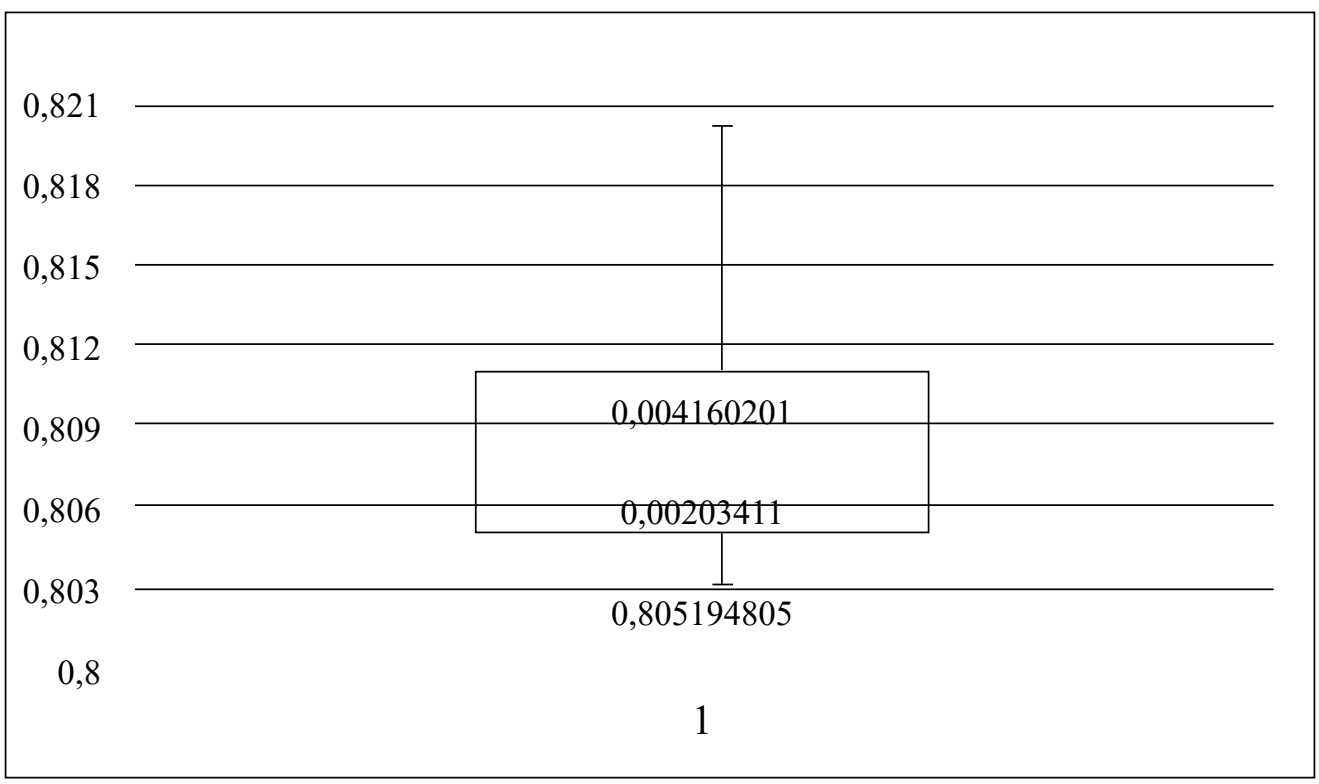

Fonte: Os autores (2017)

Com isso, pôde-se observar o comportamento dos coeficientes e concluir que a distribuição ocorre de forma relativamente homogênea e com pouca variação em torno da mediana.

Na sequência, nota-se que, ao se comparar as características das duas faixas ortogonais do caso 2 da tabela de Marcus que, embora de mesmas dimensões, há diferença na intensidade do deslocamento e o local onde ele é mais acentuado. Esse valor torna-se maior na viga com dois apoios simples, sendo de $2 \mathrm{~mm}$, enquanto na viga com um apoio simples e um engaste é de apenas $0,8319 \mathrm{~mm}$. Dessa forma, pode-se afirmar que a viga com dois apoios simples, quando em igual dimensão, é menos rígida.

Com o objetivo de definir um coeficiente nomeado lambda $(\lambda)$, sendo este baseado na tabela 02 (comparativa entre essas duas vigas em análise), foi realizado um estudo estatístico. Nesse estudo, devido à diferença das ordens de grandeza dos comprimentos das menores vigas, que força o coeficiente a atuar num valor elevado, foi necessário eliminar os primeiros cinco valores encontrados para o lambda $(\lambda)$. Tendo em vista tal critério e com o auxílio do software Excel, obteve-se um quadro para o intervalo de confiança, expressa expresso pela tabela 6.

Tabela 6 - Intervalo de confiança para o caso 2 da tabela de Marcus

\begin{tabular}{cc}
\hline $\mathrm{N}^{\circ}$ de elementos da amostra & 156 \\
\hline DESVIO PADRÃO & 0,012081183 \\
NÍVEL DE CONFIANÇA & $95 \%$ \\
MARGEM DE ERRO & 0,001895812 \\
LIMITE INFERIOR & 0,809079855 \\
MÉDIA & 0,810975667 \\
LIMITE SUPERIOR & 0,812871479 \\
\hline
\end{tabular}

Fonte: Os autores (2017) 
Gilson de Sousa Oliveira Neto, Osmar Alexandre do Amaral Neto, Vera Lúcia da Silva, Eduardo Cesar Cordeiro Leite, Ítalo Linhares Salomão

Com base nos dados da tabela 6 obteve-se a figura 05 e a figura 6 , os quais facilitam a visualização dos resultados.

Figura 5 - Intervalo de confiança para o caso 2 da tabela de Marcus

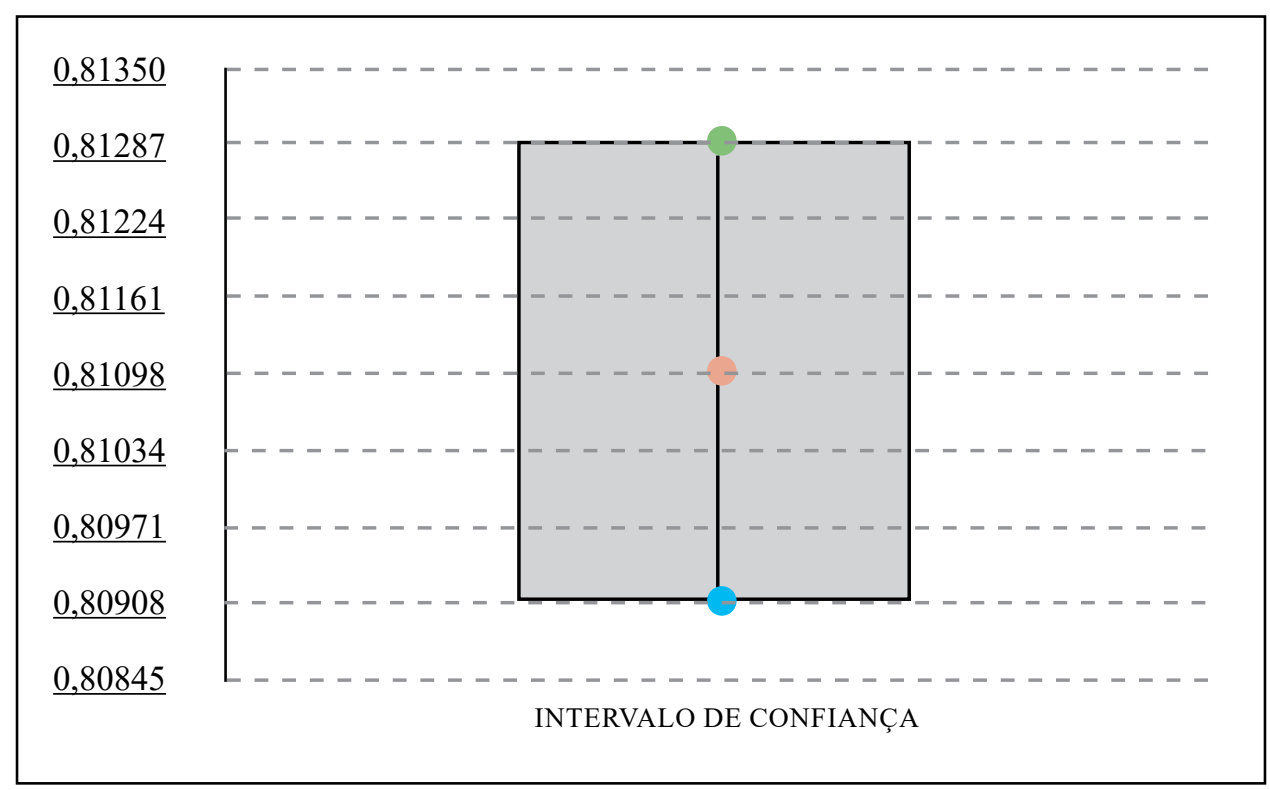

Fonte: Os autores (2017)

Figura 6 - Intervalo de confiança para o coeficiente do caso 2 da tabela de Marcus

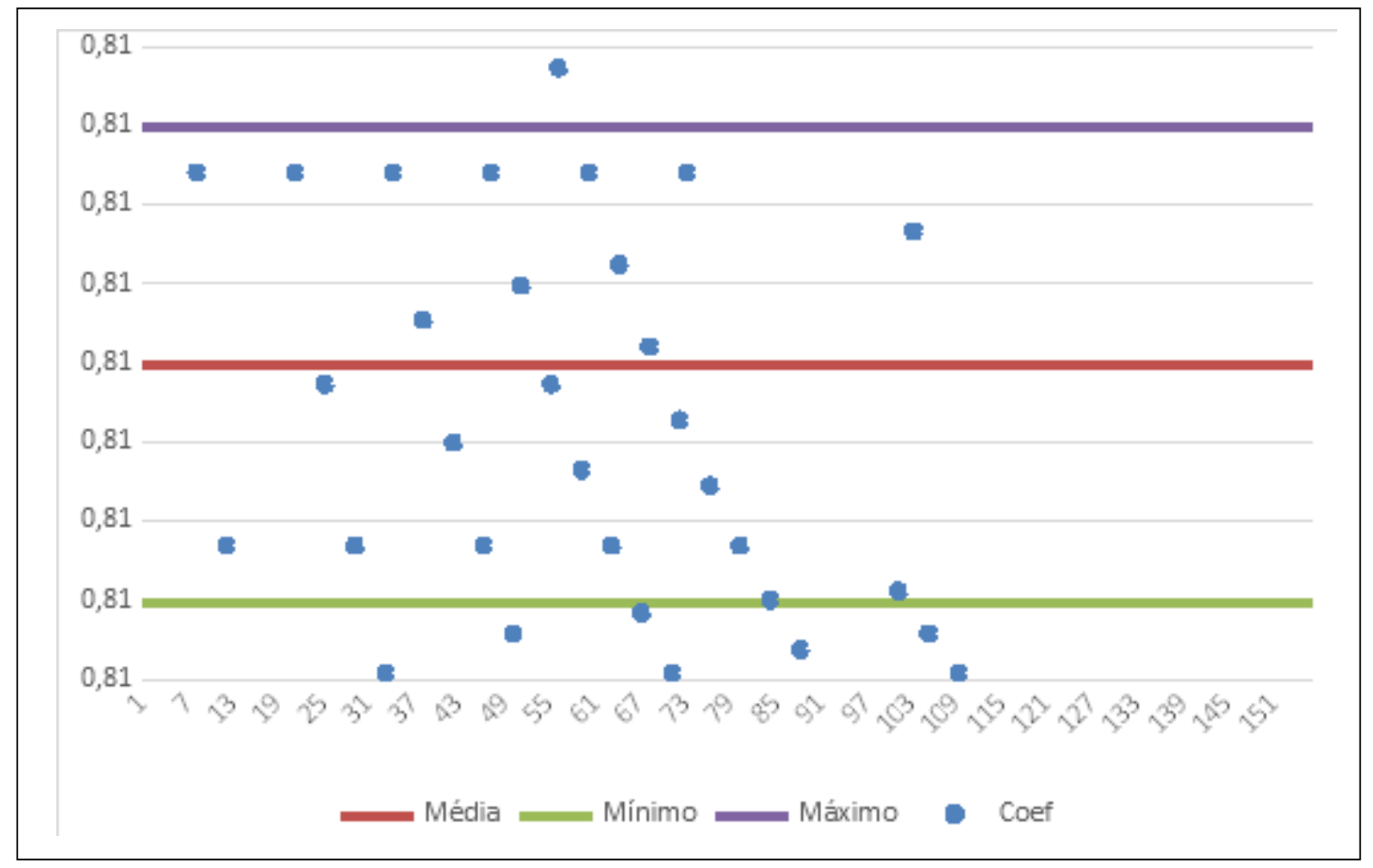

Fonte: Os autores (2017) 
Informações demonstram que um possível coeficiente para o lambda $(\lambda)$ está entre 0,809079855 e 0,812871479 , atuando numa "zona de confiabilidade maior" no valor de 0,810975667 , com margens de erro de 0,001895812 para mais ou para menos.

Para o caso 4, foi calculada a estatística descritiva expressas na tabela 7 e na figura 7.

Tabela 7 - Estatística descritiva caso 4

\begin{tabular}{cc}
\hline Média & 0,675693893 \\
\hline Erro padrão & 0,000974847 \\
Mediana & 0,672131148 \\
Modo & 0,672727273 \\
Desvio padrão & 0,011072137 \\
Variância da amostra & 0,000122592 \\
Curtose & 19,6924116 \\
Assimetria & 3,958634906 \\
Intervalo & 0,081210191 \\
Mínimo & 0,668789809 \\
Máximo & 0,75 \\
CV & $1,639 \%$ \\
Contagem & 129 \\
\hline
\end{tabular}

Fonte: Elaborada pelo Autor

Figura 7 - Box Plot caso 4

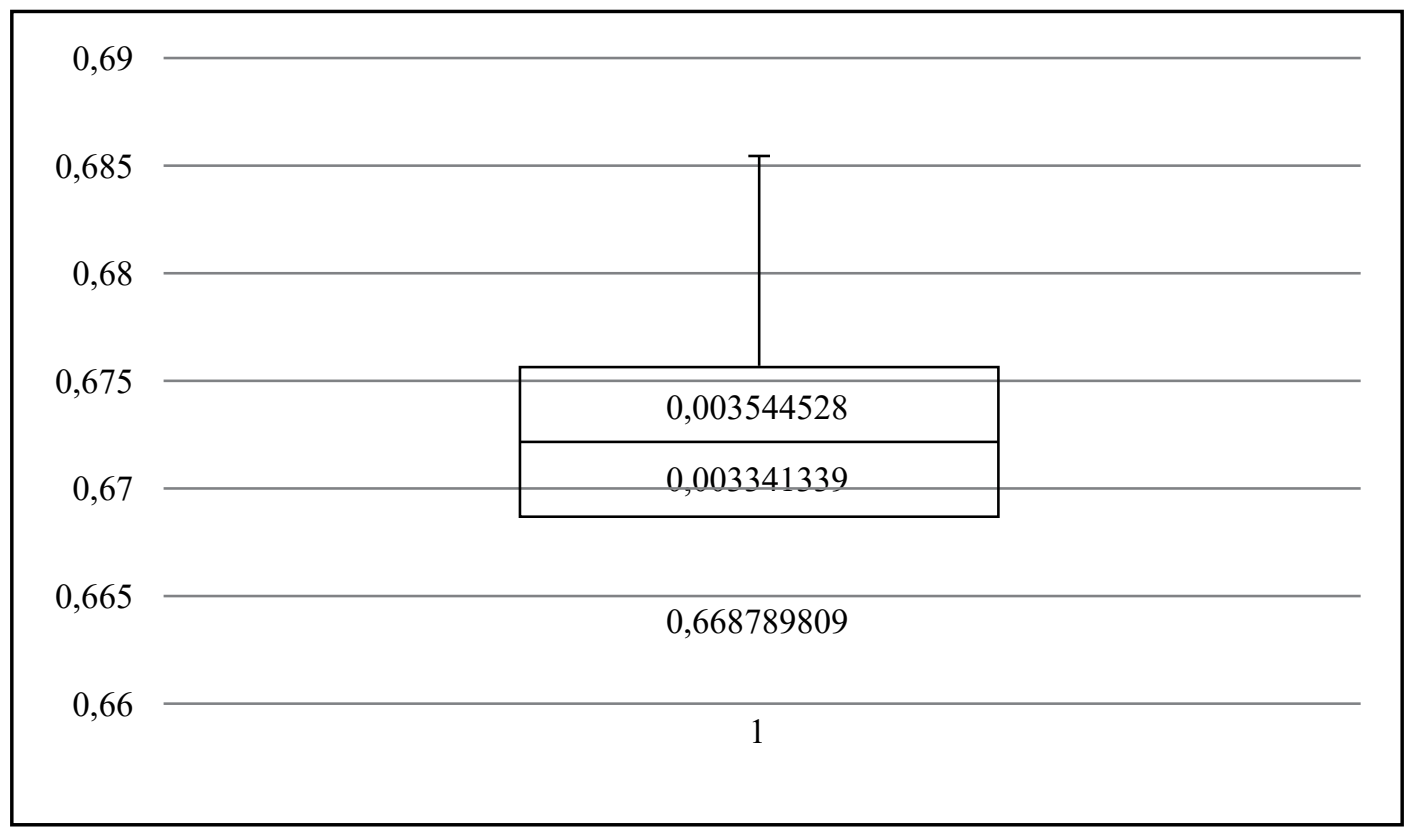

Fonte: Os autores (2017)

Com isso, pôde-se observar o comportamento dos coeficientes e concluir que a distribuição ocorre de forma bem homogênea, com uma variação menor em torno da mediana que no caso anterior.

Prosseguindo, nota-se que, ao comparar as características das vigas do caso 4 da tabela de Marcus, apesar de terem as mesmas dimensões, há diferença na intensidade do deslocamento, mas praticamente não há variação onde ele é mais 
acentuado. Essa medida torna-se maior na viga com dois apoios simples, sendo de $2 \mathrm{~mm}$, enquanto na viga bi engastada é de apenas 0,4 $\mathrm{mm}$. Dessa forma, pode-se afirmar, baseando-se no mesmo critério do caso anterior, que a viga com dois apoios simples e com dimensões iguais é menos rígida que a com dois engastes em análise.

Orientado pela tabela comparativa entre essas duas vigas em análise, foi realizado outro estudo estatístico para se obter o coeficiente lambda. Nesse estudo, tomando-se o mesmo critério do caso anterior, foram eliminados os primeiros cinco valores encontrados para o lambda $(\lambda)$. Tendo em vista tal critério, obteve-se um quadro para o intervalo de confiança, expresso pela tabela 08 .

Tabela 8 - Intervalo de confiança para o caso 4 da tabela de Marcus

\begin{tabular}{cc}
\hline $\mathrm{N}^{\circ}$ de elementos da amostra & 129 \\
\hline DESVIO PADRÃO & 0,011072 \\
NÍVEL DE CONFIANÇA & $95 \%$ \\
MARGEM DE ERRO & 0,001911 \\
LIMITE INFERIOR & 0,673783 \\
MÉDIA & 0,675694 \\
LIMITE SUPERIOR & 0,677605 \\
\hline
\end{tabular}

Fonte: Os autores (2017)

Com base nos dados da tabela 8 , obteve-se a figura 8 e a figura 9 , as quais facilitam a visualização dos resultados.

Figura 8 - Intervalo de confiança para o caso 4 da tabela de Marcus

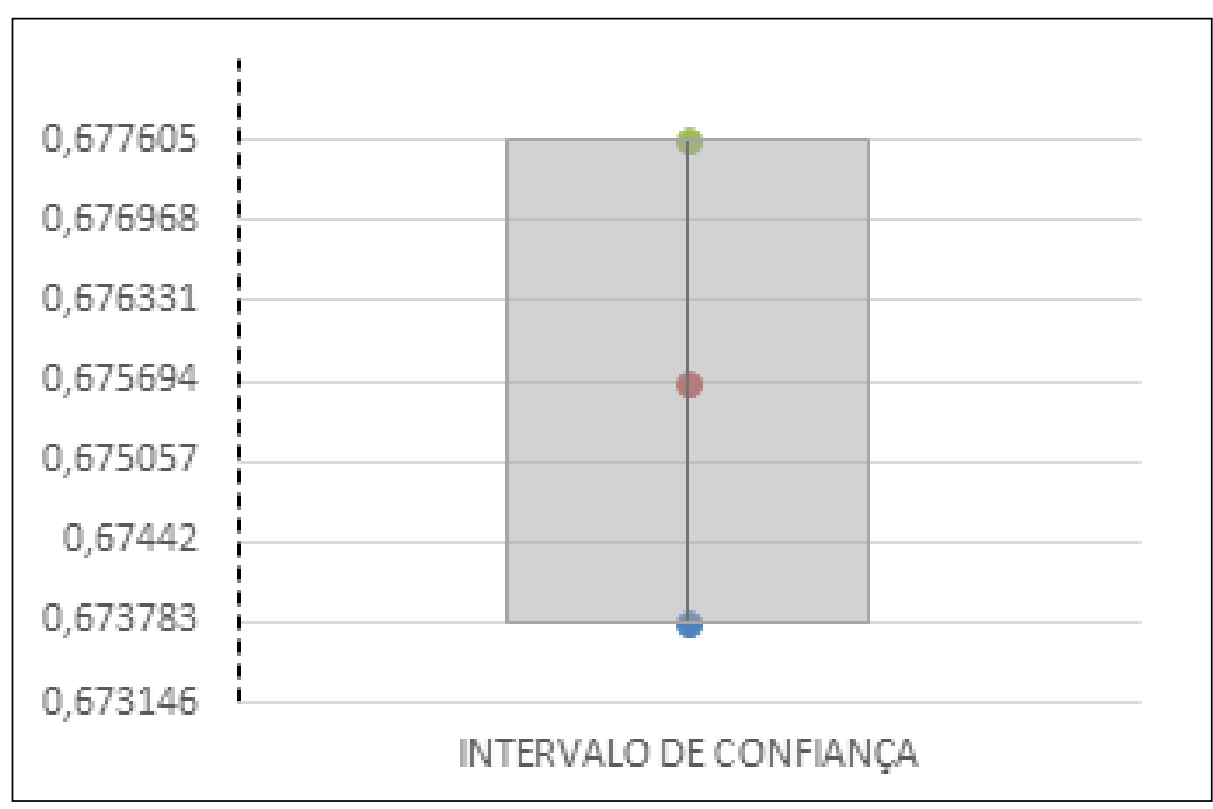

Fonte: Os autores (2017) 
Figura 9 - Intervalo de confiança para o coeficiente do 4 da tabela de Marcus

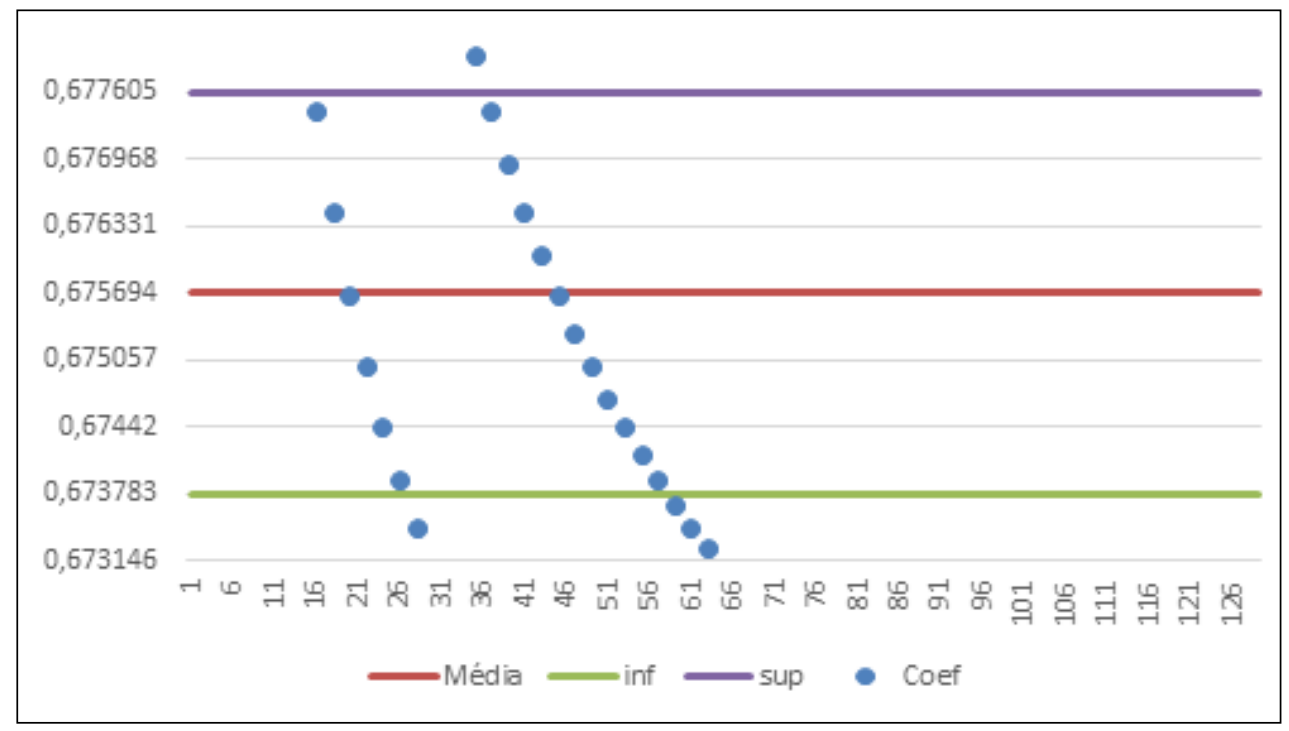

Fonte: Os autores (2017)

Estas informações demostram que um possível coeficiente para o lambda $(\lambda)$ está entre 0,673783 e 0,677605 , atuando numa "zona de confiabilidade maior" no valor de 0,675694 com margens de erro de 0,001911 para mais ou para menos.

Para o caso 5, foi calculada a estatística descritiva, expressa na tabela 9 e na figura 10.

Tabela 9 - Estatística descritiva caso 5

\begin{tabular}{cc}
\hline Média & 0,839303008 \\
\hline Erro padrão & 0,000744801 \\
Mediana & 0,836569884 \\
Modo & 0,833333333 \\
Desvio padrão & 0,009479764 \\
Variância da amostra & $8,98659 \mathrm{E}-05$ \\
Curtose & 16,38073986 \\
Assimetria & 3,646314073 \\
Intervalo & 0,066666667 \\
Mínimo & 0,833333333 \\
Máximo & 0,9 \\
CV & $1,129 \%$ \\
Contagem & 162 \\
\hline
\end{tabular}

Fonte: Os autores (2017) 
Figura 10 - Box Plot Caso 5

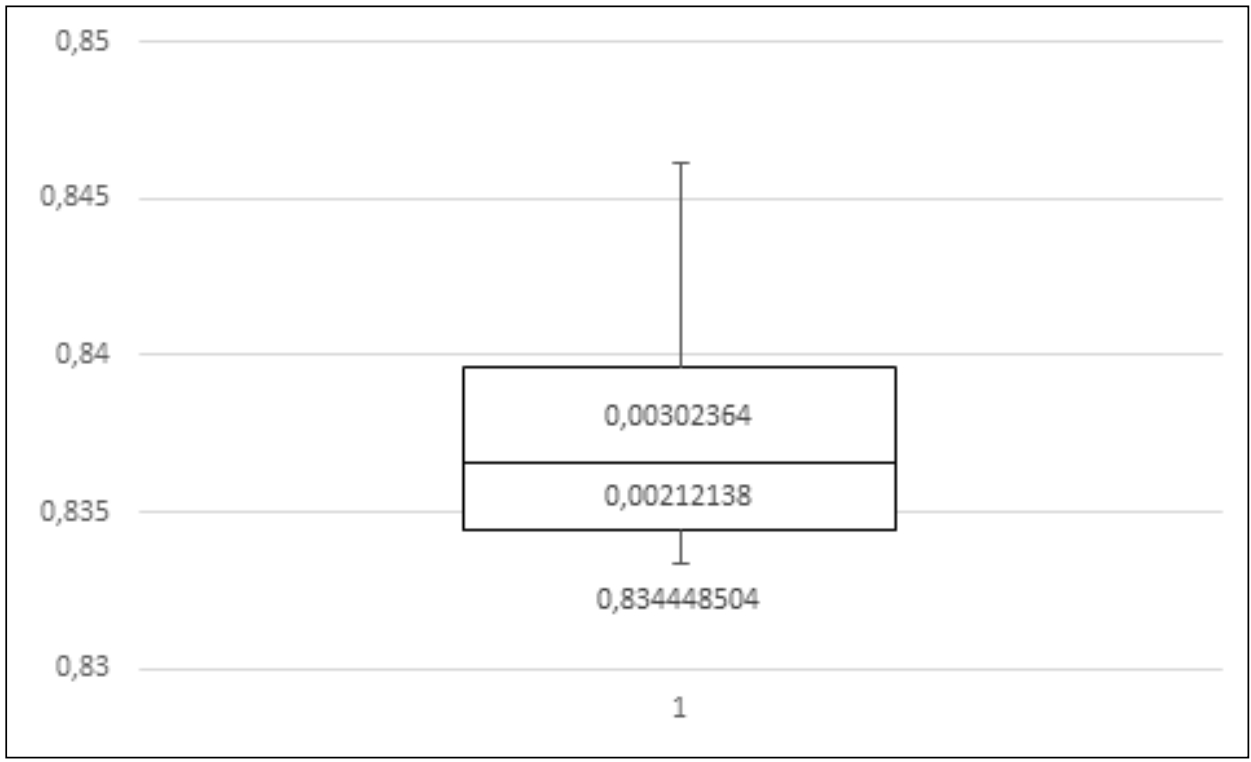

Fonte: Os autores (2017)

Com isso, pode-se observar o comportamento dos coeficientes e concluir que a distribuição ocorre de forma bem homogênea, assim como as demais, com uma variação um pouco maior que a anterior em torno da mediana e menor que no primeiro caso.

Dando seguimento, nota-se que, ao comparar as características das vigas do caso 5 da tabela de Marcus, apesar de terem as mesmas dimensões, há diferença na intensidade do deslocamento e em onde ele é mais acentuado, como no caso 2. Essa medida torna-se maior na viga com um apoio simples e o outro sendo engastado, a qual é de $0,8319 \mathrm{~mm}$, enquanto na bi engasatada é de apenas $0,4 \mathrm{~mm}$. Dessa forma, pode-se afirmar que a viga com um apoio simples e o outro sendo engastado, e com dimensões iguais, é menos rígida que a outra em análise, baseando-se no mesmo critério que as demais.

A partir da tabela comparativa entre essas duas vigas em análise, foi feito outro estudo estatístico para se obter o lambda. Nesse estudo, tomando-se o mesmo critério que os demais casos, foram eliminados os primeiros cinco valores encontrados para o lambda $(\lambda)$. Tendo em vista tal critério, obteve-se um quadro para o intervalo de confiança, expresso pela tabela 10 .

Tabela 10 - Intervalo de confiança para o caso 5 da tabela de Marcus

\begin{tabular}{cc}
\hline $\mathrm{N}^{\circ}$ de elementos da amostra & 162 \\
\hline DESVIO PADRÃO & 0,00945 \\
NÍVEL DE CONFIANÇA & $95 \%$ \\
MARGEM DE ERRO & 0,001455 \\
LIMITE INFERIOR & 0,837848 \\
MÉDIA & 0,839303 \\
LIMITE SUPERIOR & 0,840758 \\
\hline
\end{tabular}

Fonte: Os autores (2017)

Com base nos dados da tabela 10, obteve-se a figura 11 e a figura 12, a qual facilita a visualização dos resultados. 
Figura 11 - Intervalo de confiança para o caso 5 da tabela de Marcus

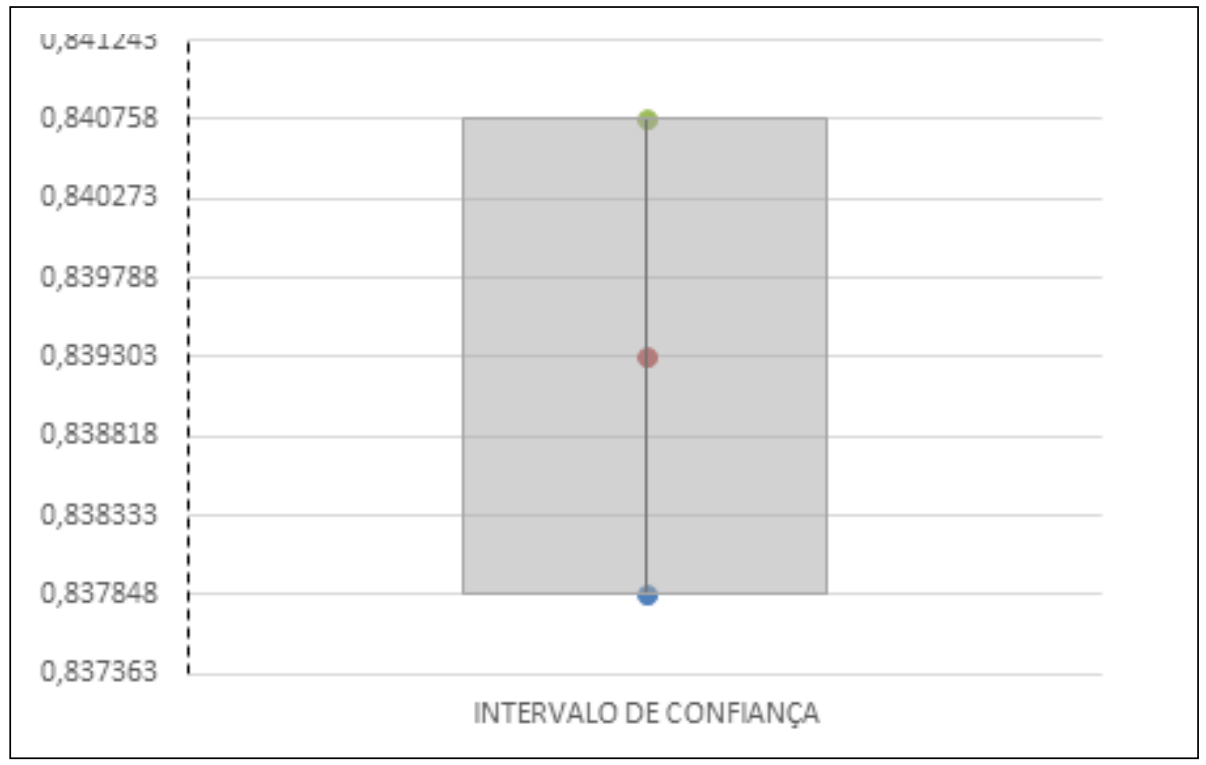

Fonte: Os autores (2017)

Figura 12 - Intervalo de confiança para o caso 5 da tabela de Marcus

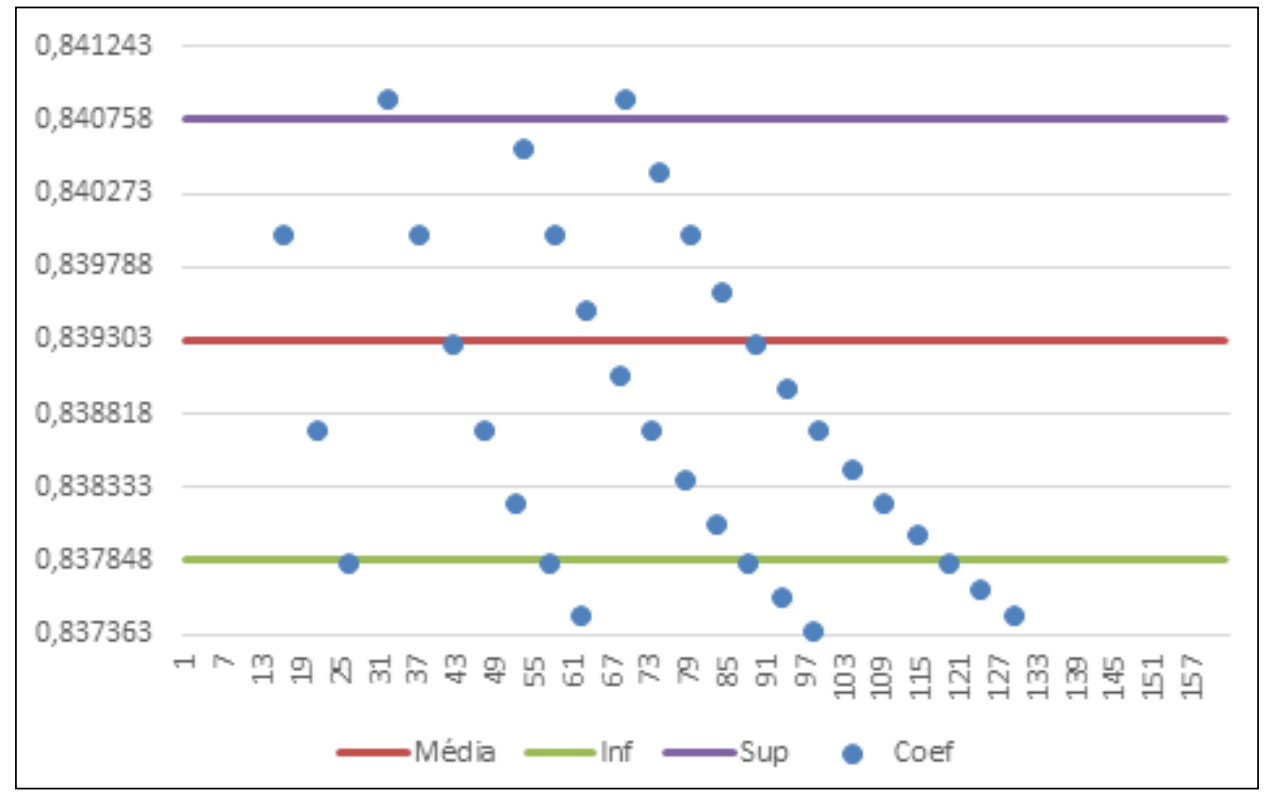

Fonte: Os autores (2017)

Estas informações demonstram que um possível coeficiente para o lambda $(\lambda)$ está entre 0,837848 e 0,840758 , atuando numa "zona de confiabilidade maior" no valor de 0,839303, com margens de erro de 0,001455 para mais ou para menos. 


\section{Conclusão}

Tendo em vista os argumentos apresentados neste artigo, conclui-se que, em síntese, analisando os conceitos da tabela de Marcus, o resultado da obtenção do coeficiente lambda $(\lambda)$ é particular para cada caso em análise, variando tanto a intensidade do deslocamento quanto o local onde ele é mais acentuado. Consequentemente, foi obtido não um valor, mas uma faixa de valores prováveis em que o parâmetro estudado $(\lambda)$ poderá ajudar engenheiros que queiram dimensionar uma laje, com $95 \%$ de precisão.

Desse modo, para o caso 2, obteve-se que a faixa de atuação para o coeficiente lambda $(\lambda)$ está entre 0,809079855 e 0,812871479, agindo numa melhor "zona de confiabilidade" no valor de 0,810975667 , com margens de erro de 0,001895812 para mais ou para menos. Assim, pode-se afirmar que, para o coeficiente, o valor de 0,810975667 é plausível de ser o mais exato.

Já para o caso 4, obteve-se que a faixa de atuação para o coeficiente lambda $(\lambda)$ está entre 0,673783 e 0,677605 , trabalhando, com maior rigor, no valor de 0,675694 , com margens de erro de 0,001911 para mais ou para menos. Com base nisso, pode-se concluir que 0,675694 é um provável valor ideal para esse coeficiente.

Por fim, para o caso 5 , obteve-se que a faixa de atuação para o coeficiente lambda $(\lambda)$ está entre 0,837848 e 0,840758 , trabalhando, com maior certeza, com o valor de 0,839303 e margens de erro de 0,001455 para mais ou para menos. Assim, o valor 0,839303 é um possível valor ideal.

Apesar de não ter sido encontrado um valor fixo (exato) para o coeficiente lambda $(\lambda)$, pode-se considerar que o objetivo deste artigo foi bem-sucedido, pois foi encontrado um intervalo confiável para possíveis valores e, de forma prática, é sugerido o uso da média geral como um possível lambda $(\lambda)$, possibilitando uma maior margem de segurança para se trabalhar.

\section{Referências}

BASTOS, Paulo Sergio dos Santos. Lajes de concreto. Bauru: UNESP, 2015.

BEER, Ferdnand P. et al. Mecânica vetorial para engenheiros. 9. ed. Porto Alegre: Bookman, 2013.

ESCOLA EDTI. O que é um BOX PLOT. Disponível em: <http://www.escolaedti.com.br/oque-e-um-box-plot/>. Acesso em: 18 nov. 2017.

HIBBELER, R. C. Resistência dos materiais. 7. ed. São Paulo: Pearson, 2010.

TRIOLA, Mario F. Introdução à estatística: atualização da tecnologia. 11. ed. Rio de Janeiro: LTC, 2014. 


\section{Gilson de Sousa Oliveira Neto}

Graduado em Engenharia Civil, Universidade de Fortaleza - Unifor

\section{Osmar Alexandre do Amaral Neto}

Graduado em Engenharia Civil, Universidade de Fortaleza - Unifor, Mestrando em Estruturas pela Pontifícia Universidade Católica do Rio de Janeiro - PUC, Coordenador do setor de comunicação da ABECE Inovação Fortaleza.

\section{Vera Lúcia da Silva}

Graduada em Estatística, Universidade Federal do Ceará - UFC, Mestre em Logística e pesquisa operacional, Universidade Federal do Ceará - UFC, Doutora em Economia, Universidade Federal do Ceará - UFC. Professora/Coordenadora de Área de Probabilidade e Estatística da Universidade de Fortaleza - Unifor.

\section{Eduardo Cesar Cordeiro Leite}

Graduado em Engenheiro Civil, Universidade de Fortaleza - Unifor, Mestre em Estruturas, Pontifícia Universidade Católica do Rio de Janeiro - PUC, Doutor em Estruturas, Universidade Stuttgart, Professor/Coordenador da Pós Graduação em Estruturas Universidade de Fortaleza - Unifor.

\section{Ítalo Linhares Salomão}

Graduado em Engenheiro Civil, Universidade de Fortaleza - Unifor, Mestre em Estruturas, Pontifícia Universidade Católica do Rio de Janeiro - PUC, Professor/Coordenador dos cursos de Engenharia Civil e Construção de Edifícios da Universidade de Fortaleza - Unifor.

Recebido em: 15.05 .2018

Avaliado em: 20.08.2018

Aceito em: 22.08 .2018 